\title{
La biblioteca escolar vista por sus usuarios: el caso de la biblioteca del CEIP Isabel la Católica (Colmenar Viejo, Madrid)
}

\author{
Linda M. FERRER \\ Catedrática de La Facultad de Documentación de la Universidad de Alcalá \\ Purificación Moscoso \\ Universidad de Alcalá. Facultad de Documentación \\ p.moscoso@uah.es
}

Recibido: junio 2011

Aceptado: noviembre 2011

\begin{abstract}
Resumen: El objeto de este estudio es evaluar la biblioteca del Centro de Educación Infantil y Primaria Isabel La Católica a través de la visión que aportan sus usuarios, desde alumnos de cinco a doce años hasta los profesores del centro. Para conocer su opinión y percepción se ha llevado a cabo una evaluación subjetiva basada en los modelos ServQUAL y en su derivado para bibliotecas LibQUAL+, que se han adaptado a las peculiaridades y características de los distintos tipos de usuarios, así como los objetivos de las bibliotecas escolares de la Comunidad de Madrid.
\end{abstract}

Palabras clave: Biblioteca escolar; evaluación subjetiva; LibQUAL+; ServQUAL; alfabetización informativa; sistemas de gestión de calidad; colegio público Isabel La Católica.

\begin{abstract}
The purpose of this paper is to evaluate the Library of the School Isabel La Católica through the vision provided by their users, including 5-12 year old students as well as teachers. Their views and perceptions have conducted a subjective evaluation based on the ServQUAL and LibQUAL+ models, that have been adapted to the characteristics of different types of users and to the objectives of the school libraries in the Region of Madrid.
\end{abstract}

Keywords: School library; assesment; LibQual+; ServQUAL; information literacy; quality management; Isabel La Católica public school.

\section{INTRODUCCIÓN}

La actual sociedad del conocimiento, una sociedad en red, ha abierto una nueva brecha entre los ricos y los pobres en información. Es claro que no es suficiente con que la información esté disponible desde cualquier lugar del planeta, y tampoco que las TICs garanticen un acceso inmediato y pertinente. Los ciudadanos necesitan un aprendizaje específico sobre cómo acceder y usar la información, lo que requiere nuevas formas de alfabetización que permitan aprovechar al máximo el potencial de la Red. 
Las bibliotecas escolares constituyen el primer eslabón de una cadena que desempeña un papel fundamental en el proceso de alfabetización informativa de la sociedad, ya que su cometido es cubrir la etapa inicial en el uso de la información. Atendiendo a las recomendaciones de la UNESCO, dos de las funciones más importantes de una biblioteca escolar son, por una parte, ofrecer oportunidades de crear y utilizar la información para adquirir conocimientos, comprender, desarrollar la imaginación y entretenerse. Por otra, enseñar a los estudiantes las habilidades para evaluar y utilizar la información en cualquier soporte, formato o medio (Pemmer, T.; Willar, G., 2002).

Asimismo, la teoría educativa que subyace en la legislación y en los modelos vigentes promueve la actividad que desarrollan los propios alumnos y enfatiza la necesidad de desarrollar estrategias para facilitar el aprendizaje autónomo, aprendizaje en el que la biblioteca desempeña un papel fundamental. El sistema educativo debe contribuir, asimismo, a formar lectores competentes, a promover actitudes reflexivas y críticas ante los distintos medios de transmisión y difusión de la cultura, así como a despertar el interés por la lectura. En todo ello, también, la biblioteca escolar es un elemento clave.

En este contexto, la biblioteca escolar es espacio de acceso, uso y comunicación de información, y se constituye en un centro de recursos educativos indispensable para la formación en las primeras etapas de la educación (Camacho Espinosa, J.A., 2005). Se necesita un nuevo modelo de biblioteca escolar plenamente integrada en el proyecto educativo del centro y que cuente con los recursos necesarios y la organización para ello (Gómez Hernández, J.A. 2002).

La implantación de este nuevo modelo obliga a realizar procesos de evaluación dirigidos a lograr una mejora continua que facilite una gestión de calidad y la constitución de un espacio atractivo para sus usuarios. Para ello, es necesario conocer la opinión de los principales implicados, los alumnos, pues sólo teniéndola en cuenta tendrán éxito los esfuerzos y estrategias realizados.

\subsection{EVALUACIÓN DE BIBLIOTECAS ESCOLARES}

La evaluación puede tener dos enfoques, el objetivo y el subjetivo. El enfoque objetivo se basa en hechos, estadísticas y datos documentados de los procesos y servicios. El subjetivo, por el contrario, se fundamenta en la percepción de los usuarios, que se mide a través de encuestas, entrevistas y cuestionarios. Prevalece, por tanto, la opinión de los usuarios, el personal y la sociedad.

En el ámbito internacional, las investigaciones relacionadas con la evaluación de bibliotecas escolares se pueden clasificar en tres grandes grupos: estudios cuantitativos de diagnóstico, estudios que analizan el impacto de estas bibliotecas en el aprendizaje de los alumnos y estudios centrados en el diseño de herramientas de autoevaluación (Estudio de campo de las bibliotecas escolares en España, 2005). 
No son representativos los estudios cualitativos dirigidos a conocer la opinión de los usuarios.

Los estudios cuantitativos se centran en los requisitos y en el funcionamiento de las bibliotecas escolares, y están diseñados para analizar de forma periódica su evolución a partir de la identificación de una serie de indicadores. Entre ellos caben destacar los trabajos realizados en Estados Unidos por el Department of Education y el National Center for Education Statistics (National Center for Education Statistics, 2004), los promovidos por el Chartered Institute of Library and Information Professionals del Reino Unido (Cilip, 2002) y los realizados por la National Library de Canadá (Duran, M.; Waltman Dashko, M., 2001).

Entre las investigaciones orientadas a investigar la incidencia de la biblioteca escolar sobre los logros en el aprendizaje de los alumnos se encuentran las fomentadas por diversos organismos de Australia (Australian Council For Educational Research, 2003) y Escocia (William, D.; Wavell, C.; Coles, L., 2001), que analizan las distintas competencias adquiridas por los alumnos.

El gobierno escocés, en la iniciativa How Good is Our School?: Self-evaluation Using Performance Indicators, ha desarrollado herramientas de autoevaluación que permiten a los centros de enseñanza realizar autodiagnósticos (Scottish Consultative Council On The Curriculum, 1999).

En España, los estudios que se han realizado constituyen un diagnóstico sobre el funcionamiento de este tipo de bibliotecas, centrados, principalmente, en los distintos ámbitos autonómicos (Ortiz-Repiso, V.; Camacho Espinosa, J.A, 2004; Gobierno De Navarra, 1999; Diputación De Barcelona, 1990; Baró, M.; Mañá, T.; Comalat, M., 2002). Referidos al ámbito nacional destacan dos, el promovido en 1997 por el Ministerio de Cultura (Anabad, 1997) y el Estudio de campo de las bibliotecas escolares en España de 2005, que representa una radiografía completa del funcionamiento de estos centros durante el curso 2004-2005.

El objeto de este estudio se centra en analizar el funcionamiento de una biblioteca escolar concreta, la del Centro de Educación Infantil y Primaria (CEIP) Isabel La Católica, a través de la visión que aportan sus usuarios, los alumnos y profesores del Centro. Se trata, por tanto, de una evaluación cualitativa, con un enfoque subjetivo, que se basa en los modelos ServQUAL y en su derivado para bibliotecas LibQUAL+.

El modelo ServQUAL (Service Quality) fue desarrollado por tres investigadores del área de marketing, los profesores Parasuraman, Berry y Zeithaml. Este modelo se fundamenta en la teoría de los Gaps -The Gaps Models of Service Quality- que explica las diferencias entre las expectativas de los clientes y aquello que realmente obtienen del servicio utilizado (Zamudio Igami, M.; Sampaio, M.I. y Vergueiro, W., 2005).

El modelo LibQUAL + fue desarrollado por un consorcio de 12 bibliotecas americanas junto con la Association of Research Libraries (ARL), que lo define como un conjunto de herramientas y servicios que las bibliotecas pueden usar para 
solicitar, procesar, entender y actuar de acuerdo con la opinión que los usuarios tienen de los servicios que ofrece (Fushimi, M., 2008). LibQUAL+, al igual que el protocolo ServQUAL, trabaja con un conjunto de dimensiones aplicables a los servicios bibliotecarios para medir el grado de satisfacción de los usuarios (Játiva Miralles, M.V; Gallo León, J.P., 2006).

\subsection{LA BIBLIOTECA DEL CEIP ISABEL LA CATÓLICA}

El colegio público Isabel La Católica se encuentra en el centro histórico del núcleo urbano de Colmenar Viejo, en la Comunidad de Madrid. En él, 14 profesores fijos imparten docencia a 181 alumnos entre los 3 y los 12 años. Los alumnos de otras nacionalidades representan el 31\% entre los de Educación Infantil y el $61 \%$ entre los de Primaria.

Desde abril de 2006 el colegio tiene una nueva biblioteca integrada en la Red de Bibliotecas escolares de la Comunidad de Madrid. De la gestión de su personal se encarga la Subdirección General de Bibliotecas, que organiza anualmente la selección para el Plan de bibliotecas escolares a través del Servicio Nacional de Empleo. Los bibliotecarios son contratados por ocho meses -de octubre a mayo-, sin que exista la posibilidad de que su contrato se prorrogue, por lo que cada curso escolar los centros tienen un bibliotecario nuevo.

La distribución de la colección se muestra en las tablas I y II.

Tabla I. Distribución de la colección por categorías

\begin{tabular}{cccccc|}
\hline Infantil & $\begin{array}{c}\text { General y } \\
\text { adultos }\end{array}$ & Juvenil & Prelectores & Otros & Total \\
\hline 3937 & 68 & 270 & 223 & 4 & 4502 \\
\hline
\end{tabular}

Tabla II. Distribución de la colección según el soporte

\begin{tabular}{ccccccccc} 
CD & CDR & DVD & Folletos & Libros & $\begin{array}{c}\text { Material } \\
\text { cartográfico }\end{array}$ & VHS* & Otros & Total \\
\hline 71 & 72 & 146 & 0 & 4210 & 23 & 54 & 0 & 4576 \\
\hline
\end{tabular}

La biblioteca está automatizada mediante el Sistema Integrado de Gestión Bibliotecaria AbsysNET. Está dotada de un puesto informático para uso del bibliotecario y otro de consulta al OPAC. Además, cuenta con una pantalla de televisión de plasma de 42 pulgadas y un reproductor de DVD.

Los usuarios activos registrados en la biblioteca son 197, de los que 181 son alumnos y 16 profesores, 2 de ellos temporales.

El horario de la biblioteca es de $11,30 \mathrm{~h}$ a $14,00 \mathrm{~h}$ y de $14,00 \mathrm{~h}$ a $18,00 \mathrm{~h}$. Durante la mañana está abierta sólo para los profesores, a los que atiende la docente res-

\footnotetext{
${ }^{1}$ Sin catalogar
} 
ponsable de la biblioteca. Por la tarde, la biblioteca se abre a todos los usuarios, que son atendidos por una bibliotecaria. La actividad de la biblioteca está organizada de manera que cada clase pueda pasar, como mínimo, una sesión a la semana en la biblioteca, donde se realizan todo tipo de actividades.

\section{METODOLOGÍA}

Para conocer la percepción de los usuarios se ha llevado a cabo una evaluación subjetiva basada en los modelos ServQUAL y en su derivado para bibliotecas LibQUAL+. Del primero se han tenido en cuenta los elementos relacionados con los servicios. Del segundo los referidos a la atención al usuario.

Se ha diseñado una encuesta que tiene en cuenta las dimensiones que plantean estos dos modelos, si bien se ha adaptado, en primer lugar, a las características del centro y de los encuestados, desde alumnos de cinco a doce años, hasta los profesores. Se ha adaptado, también, a los objetivos de las bibliotecas escolares de la Comunidad de Madrid, que es la red a la que pertenece el centro.

Se ha elaborado una encuesta principal para los alumnos y una encuesta complementaria para los profesores, con el objetivo de relacionar las respuestas y obtener una visión más completa. Para diseñarlas se ha consultado a los profesores, a fin de recabar sus opiniones y conocimientos pedagógicos.

La encuesta recoge 38 preguntas semicerradas, estructuradas en ocho dimensiones (Anexo I):

I. Uso de la biblioteca

II. Colección

III. Organización

IV. Préstamo

V. Atención al usuario

VI. Actividades de la biblioteca

VII. Instalaciones y equipamiento

VIII. Percepción global de la biblioteca

Respecto al lenguaje utilizado, se ha pretendido que fuera simple y directo para evitar posibles dudas o ambigüedades. Las respuestas se han acompañado de dibujos representativos y de colores llamativos que los alumnos no pudieran asociar con lo que habitualmente relacionan con "buen/mal comportamiento". Así, el color elegido para el "NO" es azul, y no rojo, para evitar que los niños lo rechazaran por asociarlo con una mala conducta. El color elegido para el "SÍ'" es fucsia, y no verde, que suelen identificarlo con una buena conducta.

Las mayoría de las respuestas siguen una escala valorativa con tres posibilidades: "sí', "no" e "intermedia" o "neutral" (para, por ejemplo, referirse a "a veces", "me da igual", "algunas", etc.). En otros casos se trata de repuestas variables y/o múltiples. También en algunas preguntas se ha dado la opción de responder de forma abierta, mediante la opción "otros". 
El estudio se ha llevado a cabo al final del curso escolar, a fin de que los usuarios pudieran tener una opinión formada en la que fundamentar sus respuestas.

En la encuesta han participado todos los profesores fijos y todos los alumnos, excepto los de primero y segundo de Infantil ( 3 y 4 años), ya que a juicio de los profesores los niños de esas edades todavía no han desarrollado la capacidad de opinar libremente por miedo a la represión. Se ha incluido a los niños de tercero de Infantil por dos razones: son los alumnos que más usan la biblioteca $\mathrm{y}$, en este caso, conforman un grupo muy despierto.

Los alumnos han realizado la encuesta en sus aulas dentro del horario lectivo. Los de Infantil y primero de Primaria necesitaron 2 sesiones para completarla, debido a que, por su extensión, se consideró que con una única sesión sería difícil que mantuvieran la atención y concentración necesarias.

Asimismo, se ha llevado a cabo un análisis comparativo entre las respuestas dadas por los alumnos y las de los profesores. Para el análisis y la interpretación de los datos se ha agrupado a los alumnos por ciclos por dos razones. La primera, porque cada ciclo constituye un tipo de usuario diferenciado, y es necesario conocer los intereses de cada uno de ellos a fin de poder diferenciar políticas. La segunda porque la biblioteca tiene sus fondos organizados de esta manera.

\section{ANÁLISIS E INTERPRETACIÓN DE RESULTADOS}

El índice de respuesta ha sido del 57\% en el caso de los profesores -de 14 encuestados han respondido 8- y del 78\% en el de los alumnos -de 157 encuestados han respondido 123- .

La razón por la que algunos alumnos no realizaron la encuesta se debe a que no se encontraban en el aula en el momento fijado para hacerla, que según se convino con los profesores fue en el horario de tarde, cuando muchos no regresan al colegio. El absentismo escolar es uno de los principales problemas a los que se enfrenta el centro, ya que aumenta cada año.

Con el fin de mejorar el nivel de respuesta, se les facilitó a los profesores varios ejemplares para que los alumnos que no hubieran asistido a la sesión tuvieran la oportunidad de participar en la encuesta. Se recogieron algunas en el buzón habilitado al efecto, pero aún así el $22 \%$ de los alumnos no la hizo.

\subsection{USO DE LA BIBLIOTECA}

La mayoría de los usuarios utiliza la biblioteca entre una y dos veces al mes (63\% de los alumnos y $62 \%$ de los profesores). Sólo el $21 \%$ de los alumnos y el $25 \%$ de los profesores viene todas las semanas. Los que más la usan son los alumnos de Infantil (gráfico 1). 


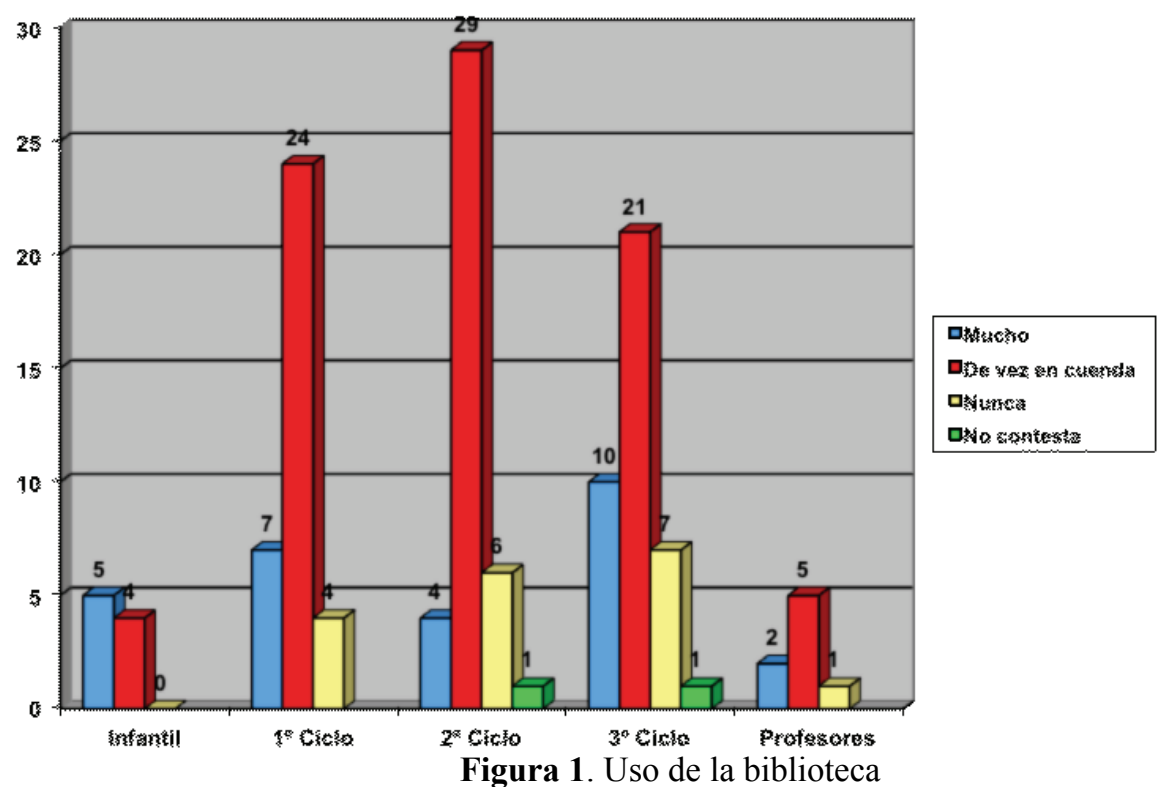

Usan la biblioteca, principalmente, para coger libros para leer en casa -la respuesta mayoritaria- y para realizar las actividades que hace la biblioteca. Van poco para estudiar, leer o jugar (gráfico 2). No existe la posibilidad de que la utilicen para acceder a Internet porque el único ordenador que hay no tiene conexión, es sólo para consultar el OPAC.

Figura 2. Para qué usan los alumnos la biblioteca

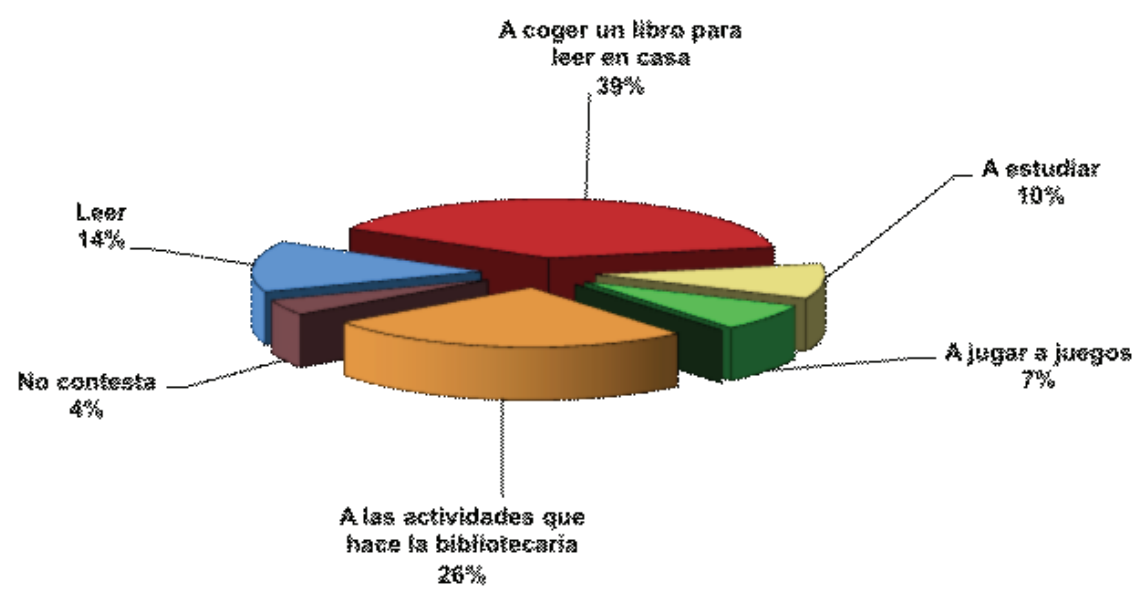


Respecto a las razones por las que no van a la biblioteca, no se aprecian diferencias significativas entre las distintas edades. La respuesta mayoritaria es que "no saben qué libros coger", seguida de "no tengo carnet", lo que resulta cuanto menos sorprendente, ya que todos los alumnos del colegio lo tienen; se les hace por regla general y se les guarda en la biblioteca (gráfico 3). Es necesario, pues, reforzar la orientación a los alumnos, así como las campañas informativas y de promoción de la biblioteca ("te hago un carné", carteles y reuniones informativas sobre las actividades, etc.). Es preciso, igualmente, implicar a los profesores en ello. Preocupa, aunque sólo represente el $5 \%$ de las respuestas, que una de las razones aducidas sea que sus padres no les dejan coger libros. Se trata, por tanto, de otra de las cuestiones en la que la biblioteca y el colegio han de trabajar.

Figura 3. Por qué los alumnos no van a la biblioteca

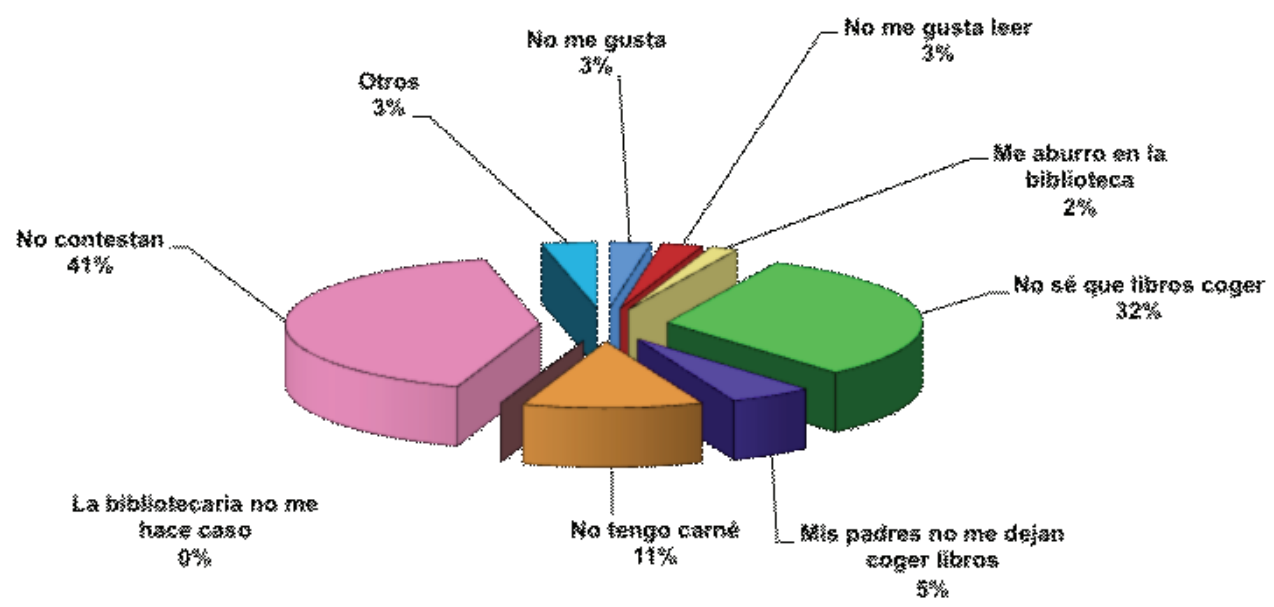

Algo más de la mitad de los alumnos usa otras bibliotecas además de la del colegio. De éstos, al $82 \%$ le gustan por igual la del colegio y las otras, el $10 \%$ prefiere las otras y el $8 \%$ la del colegio. Lo que más les atrae de las otras bibliotecas es poder pedir en préstamo CDs y películas, seguido de la atención que reciben y de que la colección sea más grande y variada (gráfico 4). Los profesores, sin embargo, no suelen ir a bibliotecas externas al centro para coger libros de apoyo a su actividad docente. 
Figura 4. Qué les gusta a los alumnos de las otras bibliotecas

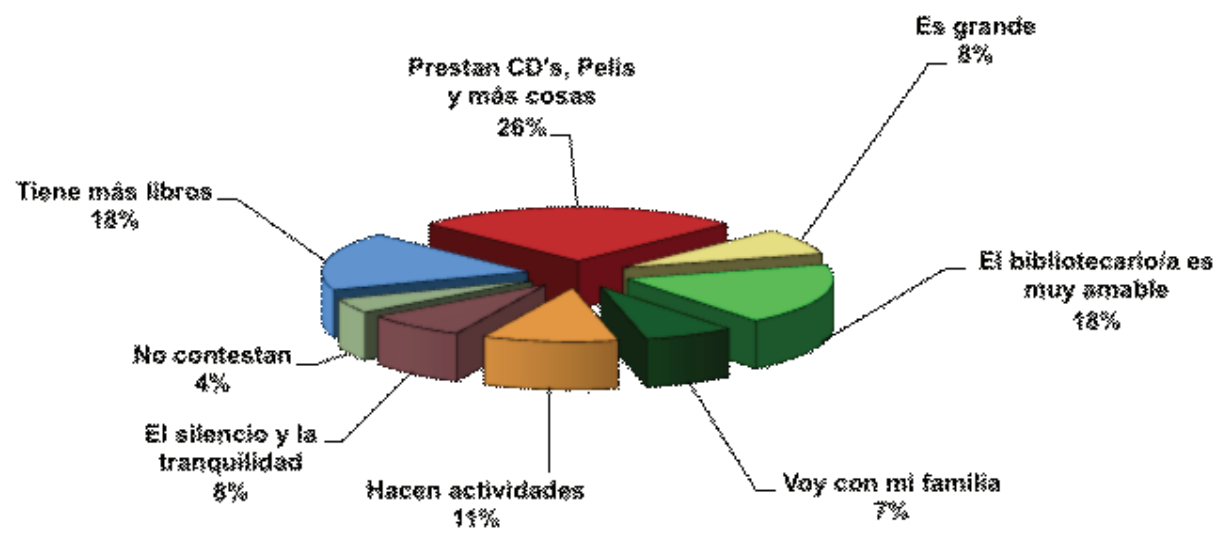

Cuando se les preguntó a los niños "qué aprenden en la biblioteca del colegio", las respuestas principales fueron "a leer", "palabras" y "a inventar cuentos" (gráfico 5). En la opción otros, un alumno contestó que en la biblioteca aprendía a reírse, y otro dijo "cuentos nuevos". Entre las respuestas de los profesores, también "aprender a leer" y "palabras nuevas" son las dos principales $(20 \%$ y $14 \%$ respectivamente). Sin embargo, ocupa el tercer lugar "cosas de clase" (14\%). La opción "otros" recoge respuestas muy diversas. Los profesores opinan que en la biblioteca los alumnos aprenden cultura, a considerar la biblioteca como recurso, a expresarse y a dramatizar, a crear actividades diferentes y a disfrutar con la lectura. Consideran también que la biblioteca hace crecer la imaginación de los niños.

Figura 5. Qué aprenden, según ellos, en la biblioteca

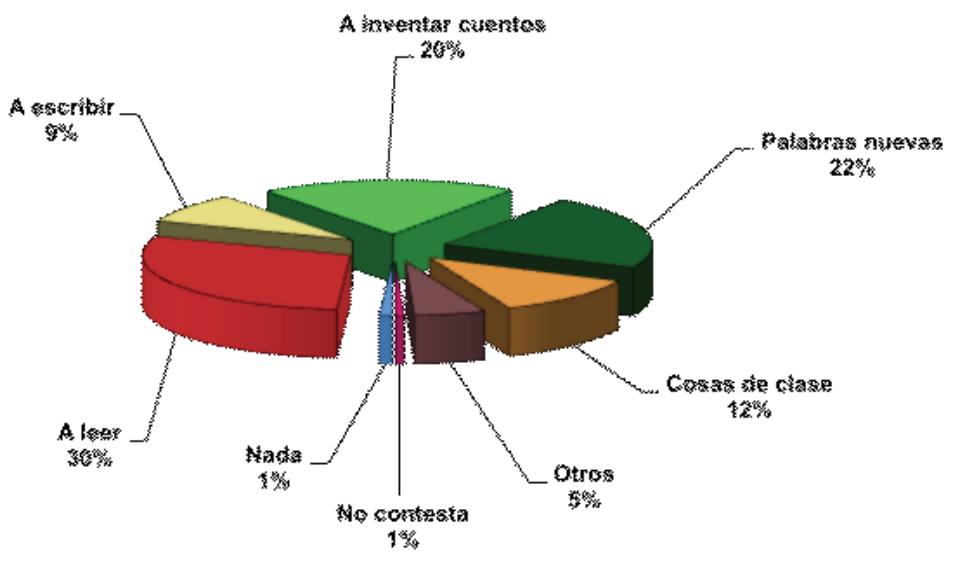




\subsection{HÁBITO DE LECTURA}

En todas las edades el hábito de lectura es alto, lo que denota un excelente trabajo por parte del colegio y de la biblioteca, especialmente si tenemos en cuenta el alto porcentaje de alumnos inmigrantes del centro.

El 55\% lee tres o más libros al mes, y casi una cuarta parte, al menos dos, lo que se corrobora con las estadísticas de préstamo, que en los últimos meses se corresponden con entre 200 y 250 libros al mes. Los alumnos que más libros leen son los de Infantil y primer ciclo de Primaria.

Sobre los libros que más les gustan, las respuestas que representan los mayores porcentajes son los libros de miedo, los de risa, los de aventuras y los de amor (gráfico 6). Si se analizan los resultados por ciclos se observa que los alumnos de Infantil prefieren los libros de risa a los de miedo, que son los más valorados por los tres ciclos de primaria, y llama la atención el interés de los alumnos del primer ciclo de Primaria por los libros de dinosaurios, de matemáticas y de religión. Los mayores se diferencian del resto por su preferencia por los libros de aventuras.

Figura 6. Qué libros les gustan más

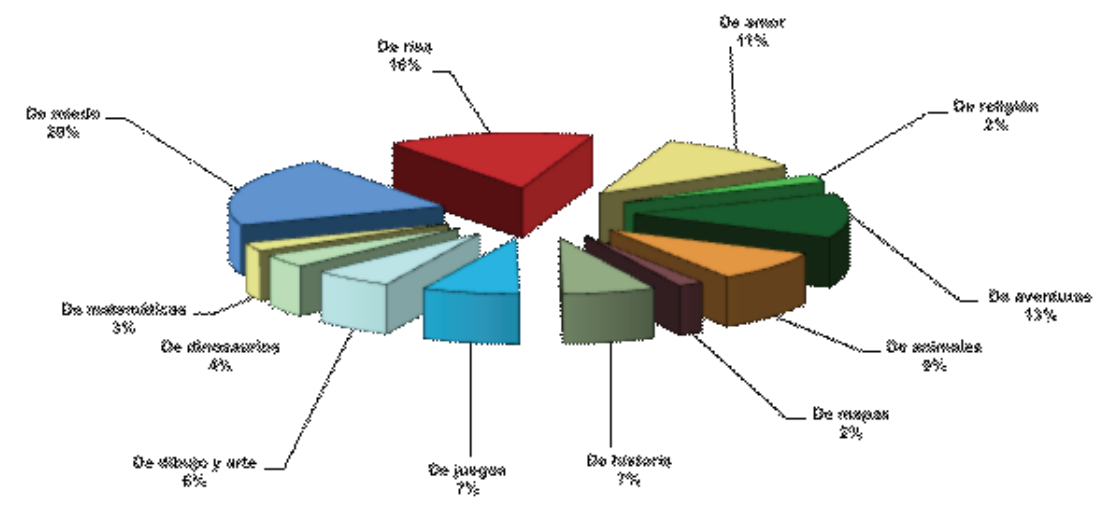

\subsection{SOBRE LA COLECCIÓN}

Al 68\% de los alumnos que realizaron la encuesta le gustan los libros de la biblioteca del colegio, si bien a casi una tercera parte sólo le gustan algunos. A los alumnos de Infantil les gustan todos. Sin embargo, al 85\% de los alumnos y al $75 \%$ de los profesores encuestados les gustaría que la colección se renovara con más frecuencia.

La política de desarrollo de la colección consiste en adquirir libros nuevos, cada curso, para Infantil y para uno de los tres ciclos de Primaria. Ahora bien, no existe un presupuesto fijo destinado a adquisiciones, por lo que las nuevas com- 
pras están supeditadas a la disponibilidad presupuestaria. Este último año, por ejemplo, no se ha podido comprar nada por falta de presupuesto.

Según la opinión de los alumnos, faltan libros de risa, de miedo, de aventuras, de amor y de juegos, principalmente (gráfico 7), resultados que coinciden, en general, con las respuestas sobre los libros que más les gustan (gráfico 6).

Figura 7. Qué libros creen que faltan en la biblioteca

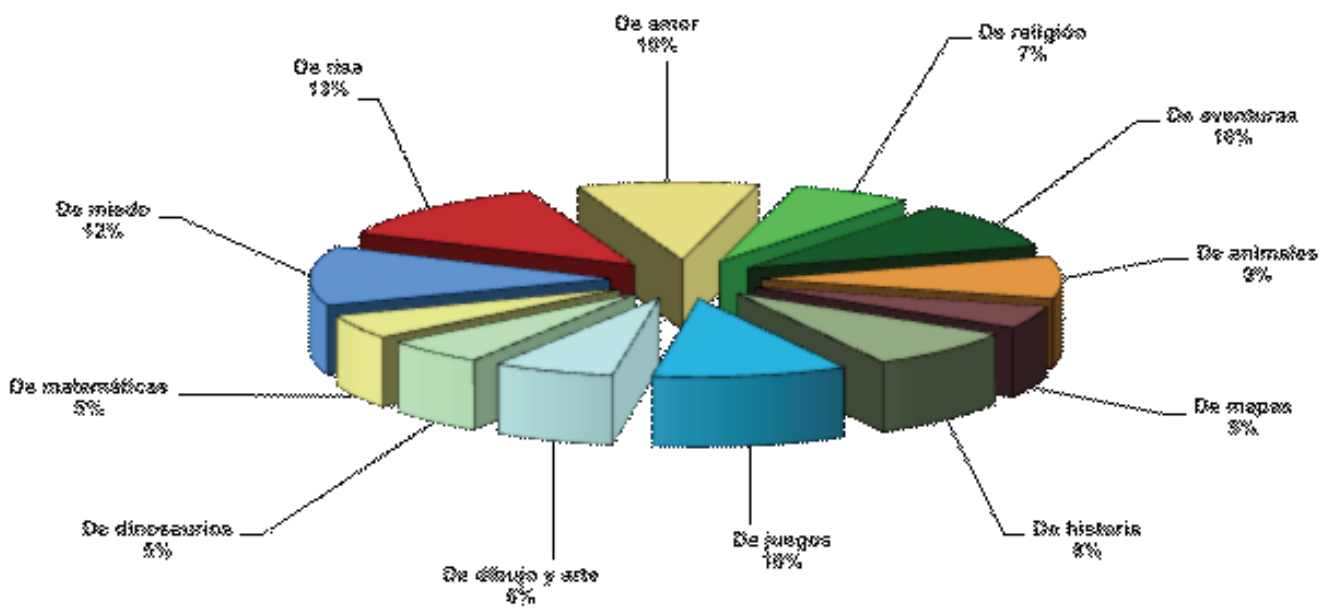

Tanto los profesores como los alumnos tienen una excelente opinión sobre el aspecto y el orden de la biblioteca. En cuanto a la ordenación de los fondos, hay que considerar que el $12 \%$ de los alumnos o no encuentra los libros que busca o no entiende cómo están ordenados. Algunos profesores consideran que el sistema utilizado es demasiado complicado para los niños. Es necesario, por tanto, incidir en la formación de los usuarios, así como colocar paneles explicativos en lugares visibles.

\subsection{SOBRE EL PRÉSTAMO}

A raíz del alto índice de robos y de la falta de cuidado en los materiales prestados, se adoptó como medida temporal la exclusión del préstamo de todos los materiales no librarios. Sin embargo, a casi el $80 \%$ de alumnos le gustaría poder pedir en préstamo materiales audiovisuales. Varios profesores no comparten esta opinión pues consideran que son demasiado pequeños para cuidarlos apropiadamente.

La política de préstamo domiciliario establece que los alumnos pueden pedir un libro a la semana. En conjunto, es muy similar el número de niños que en una semana tiene tiempo suficiente para leer un libro (46\%) y el que no $(42 \%)$. Sin embargo, la edad, en este caso, marca diferencias importantes. Hasta que llegan al 
segundo ciclo de Primaria son más a los que una semana les parece suficiente, mientras que ocurre lo contrario entre los alumnos del tercer ciclo de Primaria (gráfico 9). Esto tiene que ver, lógicamente, con las características de los libros, en los que predominan texto o ilustraciones según las edades.

Figura 8. Opinión de los alumnos sobre los plazos del préstamo domiciliario

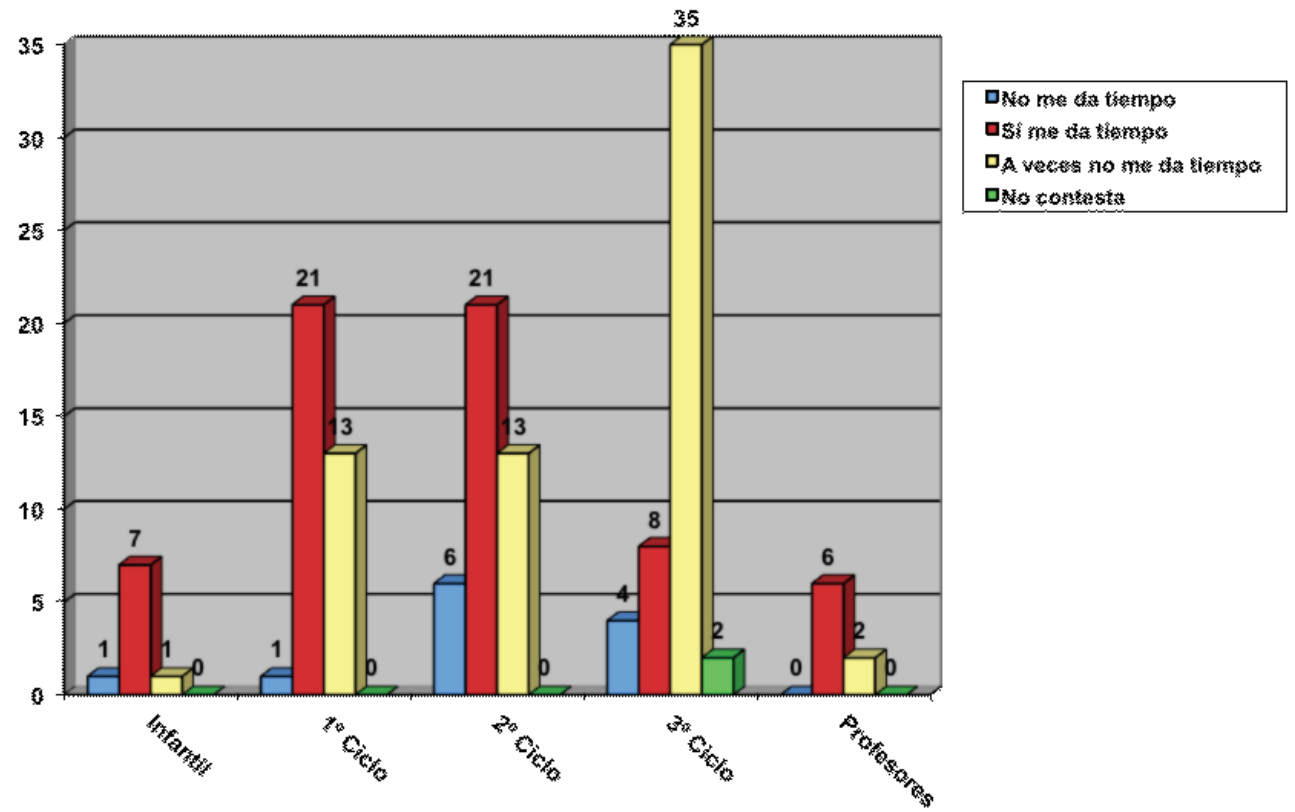

Ahora bien, con independencia de que el tiempo les parezca o no suficiente, el $84 \%$ preferiría que el plazo se ampliara, la mayoría a dos semanas, y al $75 \%$, le gustaría poder pedir en préstamo más de un libro. Los profesores, sin embargo, no comparten esta opinión, pues consideran adecuada la política establecida, tanto en lo que se refiere a los plazos como al número de ejemplares.

\subsection{SOBRE LA ATENCIÓN AL USUARIO}

El horario actual de atención a los alumnos es de 4 horas por la tarde, de 14:0018:00h, de las cuáles sólo una hora y media coincide con el horario lectivo. Al $64 \%$ de los alumnos y al $74 \%$ de los profesores les gustaría que la biblioteca estuviera abierta también por la mañana.

En general, los usuarios se encuentran muy satisfechos con la actitud y la atención que les presta la bibliotecaria. Lo están todos los profesores que contestaron la encuesta.

Para el $96 \%$ de los niños la bibliotecaria siempre les ayuda y orienta en la lectura. 
La política de personal -según la cual cada curso contratan un bibliotecario nuevo, durante los meses de octubre a mayo - no la aprueba ninguno de los profesores ni el 77\% de los alumnos encuestados, a los que les gustaría que no cambiaran de bibliotecario todos los años.

\subsection{SOBRE LAS ACTIVIDADES DE LA BIBLIOTECA}

Al $85 \%$ de los niños y al $87 \%$ de los profesores les gustan las actividades que se realizan en la biblioteca. Les gustan a todos los alumnos de Infantil y del primer ciclo de Primaria, mientras que entre los de segundo y tercer ciclo de Primaria hay un $20 \%$ a los que no siempre le agradan. La actividad mejor valorada es el cuentacuentos: representa el $20 \%$ de las respuestas de los profesores y el $22 \%$ de las de los alumnos. Las respuestas que ocupan el segundo lugar de preferencia no coinciden, en el caso de los alumnos es "el teatrillo" y en el de los profesores "la hora del cómic". En tercer y cuarto lugar los profesores prefieren la "representación teatral" y el "cuento encadenado". La tercera actividad preferida por los niños también es la "representación teatral", pero la cuarta es la visita a la biblioteca (gráficos 9 y 10).

Figura 9. Actividades preferidas por los alumnos

Figura 10. Actividades preferidas por los profesores
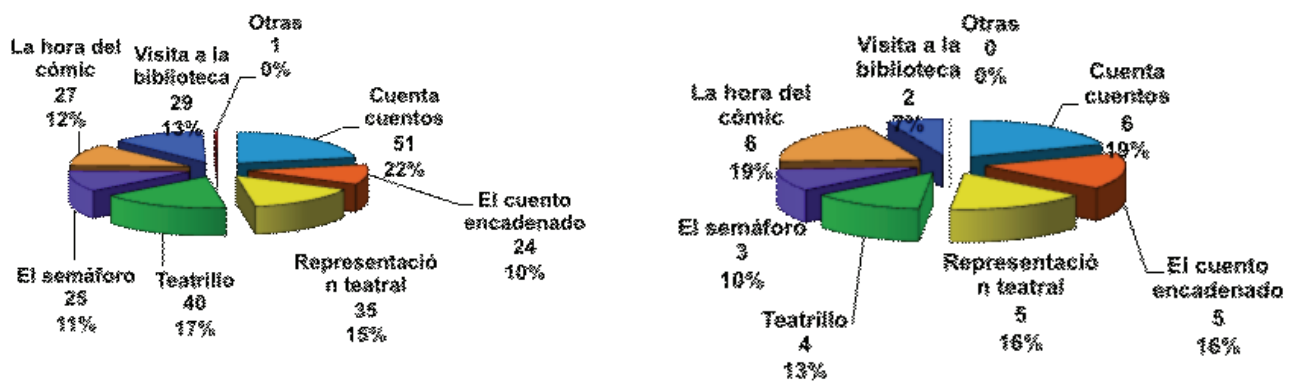

Parece pues, que, en general, si se les pregunta a los niños prefieren actividades en las que son más espectadores que protagonistas, mientras que en el caso de los profesores ocurre lo contrario.

Cuando se les pregunta qué otro tipo de actividades les gustaría que se hicieran en la biblioteca, las dos mejor valoradas son el "pop-up" y "hacemos marionetas", tanto por parte de los profesores como de los alumnos. La siguiente actividad preferida por los niños es la de "somos reporteros". mientras que los profesores prefieren los cuentos interactivos (gráficos 11 y 12).

Hay que tener en cuenta, sin embargo que se trata de una opinión basada en la explicación que se ofreció en el momento de realizar la encuesta, ya que son actividades que nunca se han realizado en la biblioteca. 
Figura 11. Qué otras actividades les gustarían a los alumnos.

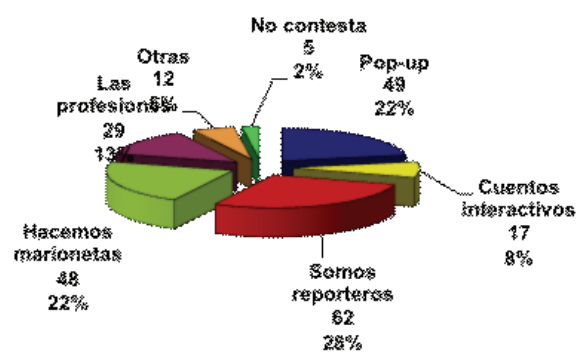

Figura 12. Qué otras actividades les gustarían a los profesores.

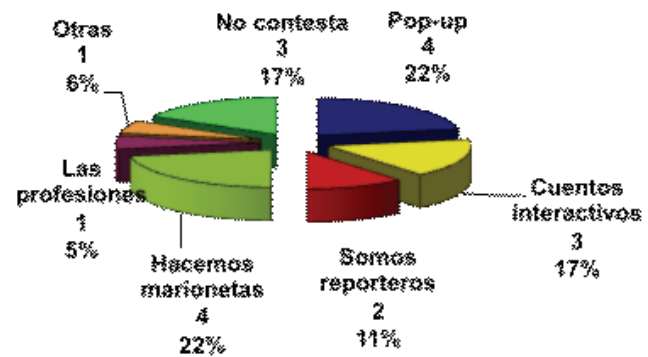

Todos los profesores encuestados creen que los alumnos deberían ir más a la biblioteca para hacer actividades. Lo mismo considera la inmensa mayoría de los niños, el 93\%.

\subsection{SOBRE EL ESPACIO Y LAS INSTALACIONES}

En conjunto, los usuarios están satisfechos con el espacio y las instalaciones de la biblioteca. Lo está el $87 \%$ de los profesores y el $79 \%$ de los niños. Entre los más pequeños es mayor el porcentaje de los que opinan que la biblioteca debería ser más grande (55\%), lo que puede deberse a que por el tipo de actividades que realizan y por su tamaño se sientan en el suelo sobre cojines que ocupan mucho espacio.

El mobiliario se renovó en 2006 y está adaptado a la edad, estatura y necesidades de los niños. Por ejemplo, los alumnos de Infantil disponen de unos cojines especiales para sentarse en el suelo. En general, a los niños las sillas y las mesas les parecen cómodas, si bien a medida que van creciendo hay más a los que no se lo parece (gráfico 13). Para el 71\% de los alumnos la altura de las estanterías es la adecuada.

Figura 13. Opinión sobre el mobiliario de la biblioteca

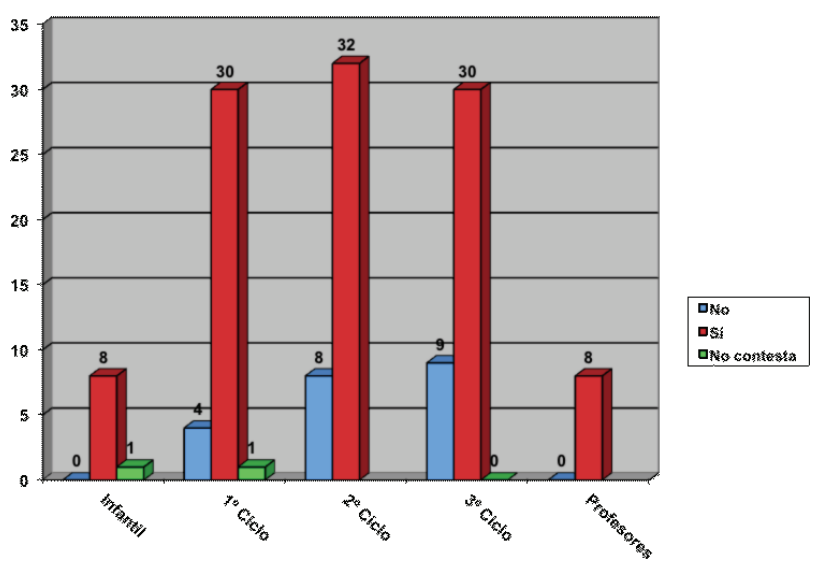


Respecto a los ordenadores, los usuarios sólo disponen de uno para consultar el OPAC, sin conexión a Internet. Este ordenador sólo lo utilizan los alumnos de segundo y tercer ciclo de Primaria, quienes consideran, en un $90 \%$, que no es suficiente, que debería haber más ordenadores en la biblioteca.

\subsection{PERCEPCIÓN GLOBAL DE LA BIBLIOTECA}

Como se observa en los gráficos 15 y 16, la inmensa mayoría de los usuarios, profesores y alumnos, valoran la biblioteca muy positivamente. En una escala del 0 al 10, el 64\% de los encuestados le pondría una puntuación entre 9-10 y el 27\% entre 7-8. La nota que le pondría el $5 \%$ es "aprobado" y sólo un $2 \%$ opina que se merece suspender. A la vista de los resultados obtenidos en las otras preguntas de la encuesta, es claro que esta opinión se basa, principalmente, en la atención que reciben, en el ambiente e instalaciones de la biblioteca y en las actividades que en ella realizan.

Figura 14. Nota de la biblioteca según los alumnos

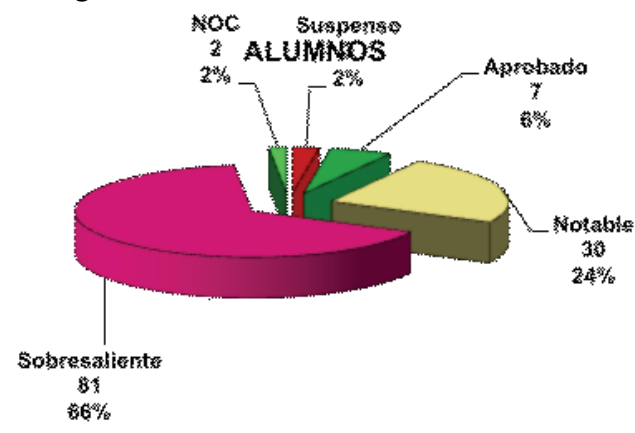

Figura 15. Nota de la biblioteca según los profesores

PROFESORES

Sumpersis

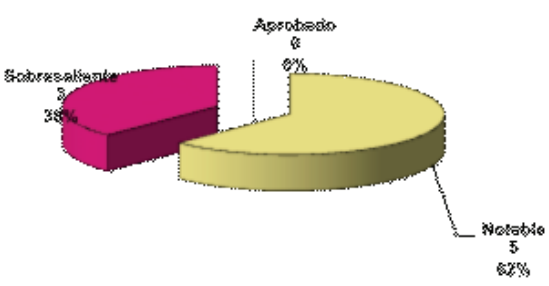

Cuando se les preguntó por lo que menos les gusta de la biblioteca, muchos optaron por no contestar -el $62 \%$ de los profesores y el $46 \%$ de los niños-. Entre los que contestaron, la respuesta más destacada es la que dejaba la opción de señalar lo que consideraran oportuno. Lo que menos les gusta a los profesores es el horario y la falta de ejemplares para las actividades de apoyo a la lectura. Los alumnos también se refirieron al horario, pero mayoritariamente expresaron que no les agrada el ruido. Otras respuestas han sido la falta de ordenadores, que no se presten películas, ni juegos ni CDs, así como la "cola para el préstamo". 
La valoración global de la biblioteca, según los distintos tipos de usuarios se muestra en el gráfico 16.

Figura 16. Valoración global de la biblioteca según los distintos tipos de usuarios

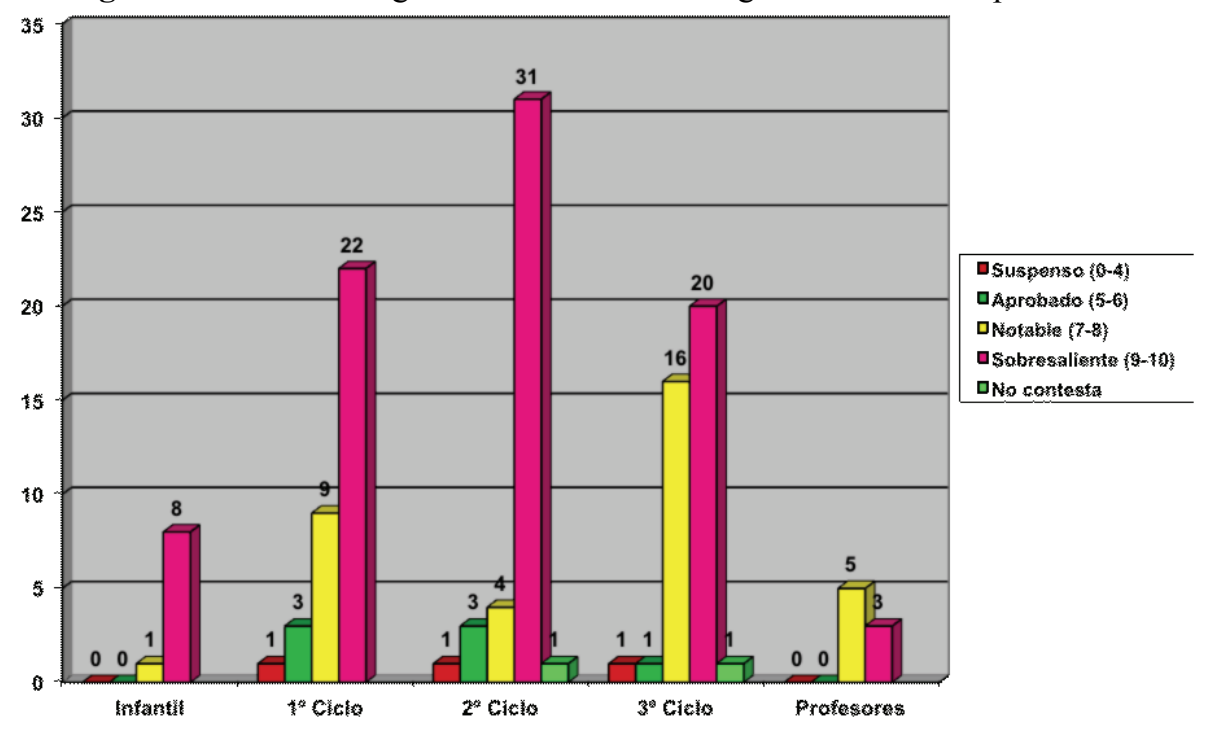

\section{CONCLUSIONES}

La colaboración de los profesores para facilitar la elaboración del estudio, así como el nivel de respuesta obtenida reflejan el interés del colegio público Isabel La Católica por la biblioteca. El interés por la lectura, que se trasluce en un alto hábito lector entre los alumnos, es consecuencia, sin duda, de la labor que realizan los docentes. Ambos hechos son un buen punto de partida para alcanzar el objetivo de la evaluación que se ha llevado a cabo: mejorar la biblioteca del centro teniendo en cuenta la percepción de sus usuarios.

La valoración global de la biblioteca es muy alta, y la opinión que tienen los usuarios es muy buena en cuanto a la atención que reciben y las actividades que se realizan. En general, están satisfechos con el espacio y con las instalaciones, a excepción de lo que tiene que ver con el equipamiento informático.

Sin embargo, el uso de la biblioteca como recurso clave para el aprendizaje no alcanza los niveles deseados, ya que son menos de la cuarta parte los alumnos que la utilizan todas las semanas, aún cuando la biblioteca está organizada para que cada clase pase al menos una sesión semanal en ella.

A la luz de los resultados obtenidos en el estudio se constata la necesidad de emprender diversas acciones. Una parte tiene que ver con el desarrollo de campañas de promoción dirigidas a los profesores, a los alumnos y a las familias. Otra ha de centrarse en un cambio de la política bibliotecaria. 
Se necesita una mayor implicación de los profesores en la utilización de la biblioteca como recurso fundamental de su docencia. Sólo de esta manera se puede lograr que los alumnos la consideren recurso básico para su aprendizaje. Establecer al menos una hora a la semana de visita dentro del horario lectivo sería un buen comienzo. Para ello, sin embargo, es necesario que la biblioteca disponga del equipamiento tecnológico idóneo.

Es preciso, además, emprender campañas de promoción entre los alumnos para incentivar su uso fuera del horario lectivo, incidiendo en la función que también desempeña la biblioteca como herramienta de ocio. La complicidad de la familia en el aprendizaje es clave, por lo que han de iniciarse acciones para reforzar el vínculo entre las familias, el colegio y la biblioteca.

Asimismo, debe incentivarse entre los alumnos la utilización de bibliotecas externas al centro, programando durante el curso visitas a las bibliotecas públicas de la zona. La biblioteca pública desempeña también una función primordial en el proceso de alfabetización informativa, por lo que su uso debe fomentarse desde la escuela.

En cuanto a los cambios en la política bibliotecaria, los principales han de centrarse en la ampliación del horario de atención al usuario, así como en las políticas de personal, préstamo, desarrollo de la colección, programación de actividades e integración de equipamiento informático.

Se considera necesario ampliar el horario de atención al usuario, y si esto no fuera posible, valorar la posibilidad de cambiarlo a horario de mañana, para así hacer coincidir las cuatro horas de trabajo del bibliotecario con las horas lectivas. Muchos profesores no acuden a la biblioteca porque el horario establecido no se adecua a la programación o al modo en que transcurre la actividad docente. Un ejemplo es el caso de primero de Infantil, en el que el horario de tarde de la biblioteca coincide con la siesta de los niños.

Uno de los cambios fundamentales está relacionado con la política de personal de la Subdirección General de Bibliotecas, que limita los contratos a ocho meses, sin posibilidad de renovación. El hecho de que cada curso escolar cambie el bibliotecario no ayuda a establecer sistemas de gestión de calidad ni a consolidar la relación entre bibliotecario y usuarios. La contratación de personal estable a jornada completa es prioritaria para mejorar el funcionamiento y el uso de las bibliotecas escolares de la Comunidad de Madrid.

Por otra parte, es necesario establecer una política de préstamo diferenciada según los distintos tipos de usuarios, ya que cada grupo tiene necesidades e intereses variados. En lo que se refiere a plazos, si tenemos en cuenta la opinión de los alumnos, el préstamo debería ampliarse de una a dos semanas para los del tercer ciclo de Primaria.

El número de ejemplares por título, así como la necesidad de disponer de más tiempo para leer los libros, no aconseja ampliar el número de libros en préstamo para los ciclos de primaria, lo que coincide con la opinión de los profesores. Am- 
pliar el tiempo de préstamo y ampliar el número de libros podría conducir a una situación de desabastecimiento. Habría que valorar, sin embargo, aumentarlo para los alumnos de Infantil, que en muchos casos devuelven el libro al día siguiente de haberlo pedido en préstamo.

Y en cuanto al préstamo de materiales audiovisuales, no hay que obviar que éste es uno de los principales atractivos para utilizar otras bibliotecas. Es cierto que este tipo de materiales exige mayor cuidado, por lo que una solución sería limitarlo a las edades en que están más capacitados para ello. Otra, enseñar a los niños cómo deben de tratarlos. Quizás se debería empezar con un programa piloto y observar los resultados.

Hacer más atractiva la colección de la biblioteca escolar implica, en primer lugar, fijar un porcentaje del presupuesto para la compra de obras nuevas todos los años, así como realizar expurgos cada cuatro o cinco a fin de renovar la imagen de la biblioteca. Una mejora fundamental pasa por tener en cuenta los intereses y preferencias de los alumnos expresados en la encuesta, que debe constituirse en un mecanismo sistemático para recabar periódicamente su opinión. Deben también considerarse las necesidades de los profesores, que demandan un mayor número de ejemplares por título para realizar las actividades de apoyo a la lectura.

En lo que se refiere a las actividades de la biblioteca, es preciso también diseñar una programación diferenciada según las distintas edades, en consonancia con las preferencias de los alumnos y con los programas didácticos de los profesores. En general, los alumnos prefieren las actividades que consisten en escuchar, como son las de animación a la lectura y formación de usuarios, en las que no son protagonistas sino espectadores. Por el contrario, los profesores prefieren las actividades de desarrollo creativo, expresión y dramatización, especialmente las de creación literaria. Ambos intereses han de conjugarse, a fin de atraer alumnos y contribuir, al mismo tiempo, a su desarrollo educativo.

Los resultados del estudio ponen de manifiesto la necesidad de efectuar más actividades de formación y orientación, que incluyen el conocimiento de las normas de la biblioteca y su organización, de las políticas de préstamo, de la búsqueda de información y del uso del OPAC.

Cabe destacar, como acción prioritaria, dotar a la biblioteca de un equipamiento informático adecuado. No vale con que el colegio disponga de un aula de ordenadores contigua. Es preciso que los niños, desde la edad más temprana, asocien biblioteca con espacio de acceso a la información y al conocimiento. Para ello es indispensable disponer de lo que en la actualidad es requisito forzoso: acceso a la Red. Enseñar a los niños las habilidades para evaluar y utilizar la información, una de las misiones de la biblioteca escolar, no puede llevarse a cabo obviando los recursos electrónicos. Hoy en día, alfabetización informativa y aprendizaje en el uso de las nuevas tecnologías van de la mano.

Los resultados del estudio corroboran la necesidad de realizar evaluaciones subjetivas en las bibliotecas escolares, a fin de conocer la opinión de sus usuarios, 
fundamentalmente la de los niños que, como se ha visto, no siempre coincide con la de sus profesores ni con las políticas de la Red a la que pertenece el centro. Sólo teniendo en cuenta sus intereses y preferencias tendrán éxito los esfuerzos y estrategias para atraerlos y hacer de la biblioteca un recurso fundamental para integrarse plenamente en nuestra sociedad, una sociedad en red.

Por último, cabe destacar, como conclusión final, que la realización de estudios de opinión debe constituirse en un mecanismo sistemático para, además, poder establecer sistemas de garantía de calidad y, de esta forma, enfocar la gestión de la biblioteca hacia la mejora continua.

\section{REFERENCIAS BIBIOGRÁFICAS}

ANABAD, FESABID y Ministerio de Cultura. Las bibliotecas escolares en España: análisis de resultados del estudio sobre su situación. Madrid: ANABAD, FESABID y Ministerio de Cultura, 1997.

AUSTRALIAN Council for Educational Research. Impact of School Libraries on Student Achievement: a Review of the Research by Michele Lonsdale. Australian Council for Educational Research, 2003.

BARÓ, M.; MAÑÁ, T; COMALAT, M. Las bibliotecas de los centros públicos de Educación Secundaria en la ciudad de Barcelona. Anales de Documentación, 2002, n. ${ }^{\circ}$ 5: 59-71.

CAMACHO ESPINOSA, J.A. La biblioteca escolar: centro de documentación, información y recursos para la comunidad educativa. Un punto de vista documental. Revista de Educación, 2005, p. 303-324.

CAMACHO ESPINOSA, J.A. Las bibliotecas escolares en Castilla-La Mancha y sus usuarios. Estudio de la situación durante el curso 2002-2003. Educación y Bibliotecas, 2003, n. ${ }^{\circ}$ 146: 118-133.

CILIP. Survey of Secondary School Libraries. Main Findings. A Report Prepared for CILIP. October 2002.

DIPUTACIÓN de Barcelona. Les biblioteques a les escoles públiques de Catalunya. Barcelona: Diputación de Barcelona, Àrea d'Educació, 1990.

DURAN, M.; WALTMAN DASHKO, M. Elementary and Secondary Schools: The Role, Challenges and Financial Conditions os School and School Library Resoruces in Canada. A Report prepared for the National Library of Canada by Michel Durand and Marla Waltmann Dashko. Culture, Tourism and the Center for Education Statistics. Canadá. September 2001.

FUSHIMI, M. Experiencia de evaluación de las bibliotecas de la UNLP: de la teoría a la práctica. En: Seminario Availaçao da Biblioteca Universitaria Brasileira San Salvador da Bahia, 29-30 de septiembre, 2008.

GOBIERNO de Navarra. Una aproximación al estudio de las bibliotecas escolares en Navarra. Claves para su mejora. Pamplona: Gobierno de Navarra. Departamento de Educación y Cultura, 1999. 
GÓMEZ HERNÁNDEZ, J. A. Gestión de Bibliotecas. Murcia: DM, 2002, p. 299320.

IFLA. Directrices de la IFLA/Unesco para la Biblioteca Escolar. IFLA, 2002.

JÁTIVA MIRALLES, M ${ }^{a}$ Victoria; GALLO LEÓN, José Pablo. LIBQUAL+TM: evaluación de calidad de servicios desde la perspectiva del usuario. Boletín de la ANABAD, 2006, vol. $56 \mathrm{n}^{\circ} 1,53-75$.

Libqual+ Charting Library Service Quality [en línea]. Washington, d.c.: Association of Research Libraries. http://www.libqual.org/

ORTIZ-REPISO, V.; CAMACHO ESPINOSA, J.A. Radiografía de las bibliotecas escolares de Castilla-La Mancha. La biblioteca escolar central. Educar en Castilla-La Mancha, 2004, 22: 3-9.

PEMMER, T., WILLAR, G. The IFLA/UNESCO School Library Guidelines. La Haya,

SCOTTISH Consultative Council on the Curriculum, 1999Taking a closer look at the School Library Resource Centre: self-evaluation using performance indicators. SCCC/HMI Audit Unit, 1999.

The status of Public and Private School Library Media Centers in the United States 1999-2000. National Center For Education Statistics., 2004. NCES 2004, p. 313.

WILLIAM, D.; WAVELL, C.; COLES, L. Impact of School Library Services on Achievement and Learning. School of Information and Media, Faculty of Management, The Robert Gordon University, 2001.

ZAMUDIO IGAMI, M.; SAMPAIO, M. I. y VERGUEIRO, W. El uso del SERVQUAL en la verificación de la calidad de los servicios de unidades de información: el caso de la biblioteca del IPEN. En: Revista Interamericana de Bibliotecología. Vol. 28, No. 2(jul-dic.2005); p. 183. 